\title{
Management of Post-Transplant Anastomotic Stricture Using Self- Expandable Metal Stent
}

\author{
Dong Wook Lee ${ }^{1}$ and Kazuo Hara ${ }^{2}$ \\ ${ }^{1}$ Division of Gastroenterology, Department of Internal Medicine, Daegu Catholic University School of Medicine, Daegu, Korea, ${ }^{2}$ Division \\ of Gastroenterology, Department of Internal medicine, Aichi Cancer Center, Nagoya, Japan
}

Anastomotic stricture (AS) is one of the complications of liver transplantation (LT) and can occur in up to $40 \%$ of living-donor LTs. Endoscopic management has become the first-line treatment of AS, and multiple plastic stents insertion has been mainly used in the past. Recently, many treatments utilizing fully covered self-expandable metal stents (cSEMSs) have been attempted, and results showing adequate treatment outcome have been reported. In this review, we look into the treatment performance and cautionary steps needed when using cSEMS as a treatment for AS. Clin Endosc 2020;53:261-265

Key Words: Constriction, pathologic; Liver transplantation; Self expandable metal stents

\section{INTRODUCTION}

Liver transplantation (LT) is a treatment method performed not only for acute liver failure but also for almost all liver diseases, including hepatocellular carcinoma and cirrhosis. ${ }^{1}$ Besides, while the number of LTs is increasing annually due to the improvement of post-transplant survival and quality of life, the imbalance of supply and demand between the donor and recipient has not yet been resolved. Recently, living-donor LT (LDLT) has been implemented as a solution to this shortcoming. ${ }^{2,3}$ However, the incidence of duct-to-duct anastomotic stricture (AS), one of the important complications occurring after LT, is known to be higher in LDLTs than in deceased-donor LTs (19\%-40\% vs. $15 \%-20 \%){ }^{4}$

Usually, endoscopic management has been the first-line therapy for AS. In particular, multiple plastic stents (MPS)

Received: April 29, 2020 Revised: May 13, 2020

Accepted: May 16, 2020

Correspondence: Kazuo Hara

Division of Gastroenterology, Department of Internal Medicine, Aichi Cancer Center, 1-1 Kanokoden, Chikusa-ku, Nagoya 464-8681, Japan

Tel: +81-52-762-6111, Fax: +81-52-764-2963, E-mail: khara@aichi-cc.jp

ORCID: https://orcid.org/0000-0002-4699-6136

(c) This is an Open Access article distributed under the terms of the Creative Commons Attribution Non-Commercial License (http://creativecommons.org/ licenses/by-nc/3.0) which permits unrestricted non-commercial use, distribution, and reproduction in any medium, provided the original work is properly cited. placement through endoscopic retrograde cholangiopancreatography (ERCP) was used. Recently, treatment using fully covered self-expandable metal stent (cSEMS) has been widely introduced. In this review, we explore AS treatment using SEMS.

\section{TYPES OF SEMS}

Traditionally, the use of uncovered SEMS in benign biliary stricture has not been recommended due to the difficulties involved in their removal. ${ }^{5}$ Therefore, for treating AS following LT, cSEMS is commonly used to ease the removal of SEMS afterward. However, due to the increased risk of stent migration in cSEMS, attempts have been made to use partially covered SEMS (pcSEMS). ${ }^{6}$ Chaput et al. reported that when pcSEMS was used in 22 patients with AS after LT, two patients suffered from bleeding and fever after removal of the pcSEMS. ${ }^{6}$ In addition, even with pcSEMS, stent migration occurred in two patients. Thus, pcSEMS is not recommended in the prevention of stent migration.

\section{REQUIRED NUMBERS OF ERCP}

In the case of AS occurred within 60 days of LT, the frequent 
causes are conditions such as post-operative edema, fibro-inflammatory response, and transient ischemia. ${ }^{7-10}$ This type of stricture has been successfully treated through MPS with one to two endoscopic dilatations. ${ }^{8}$ On the other hand, for AS occurring three months or more after LT, the plastic stent needs to be replaced by repeated ERCP, average of three to five times every eight to twelve weeks. ${ }^{11}$

However, since cSEMS has a larger diameter than plastic stents and a persistent radial force can be applied after just one ERCP, outcomes similar to that of MPS can be achieved even when one cSEMS was used. Therefore, cSEMS has the advantage of reducing the need for additional ERCP. Most studies demonstrated that patients using SEMS received an average of two ERCPs (one to place the SEMS and another to remove it). ${ }^{12}$ Fewer ERCPs are also directly related to reductions in treatment costs. Although cSEMS itself is more expensive than plastic stents, the total health care cost to treat AS is lower when using cSEMS than MPS due to lower numbers of ERCPs (in average, $\$ 8,288.50$ vs. $\$ 18,580.00, p<0.001){ }^{13}$

\section{NEED FOR STRICTURE DILATATION \& cSEMS PLACEMENT}

Selective cannulation of the bile duct is generally performed in ERCP, and a guidewire should be passed to the proximal portion of AS (Fig. 1A). To increase the success rate of stricture resolution, strictures may be dilated before cSEMS placement using devices such as balloons, Soehendra dilatation catheters or Soehendra stent retrievers (Fig. 1B) ${ }^{14}$ However, controversy exists on whether performing dilatations in treatment methods involving MPS is appropriate. ${ }^{15}$ It should also be noted
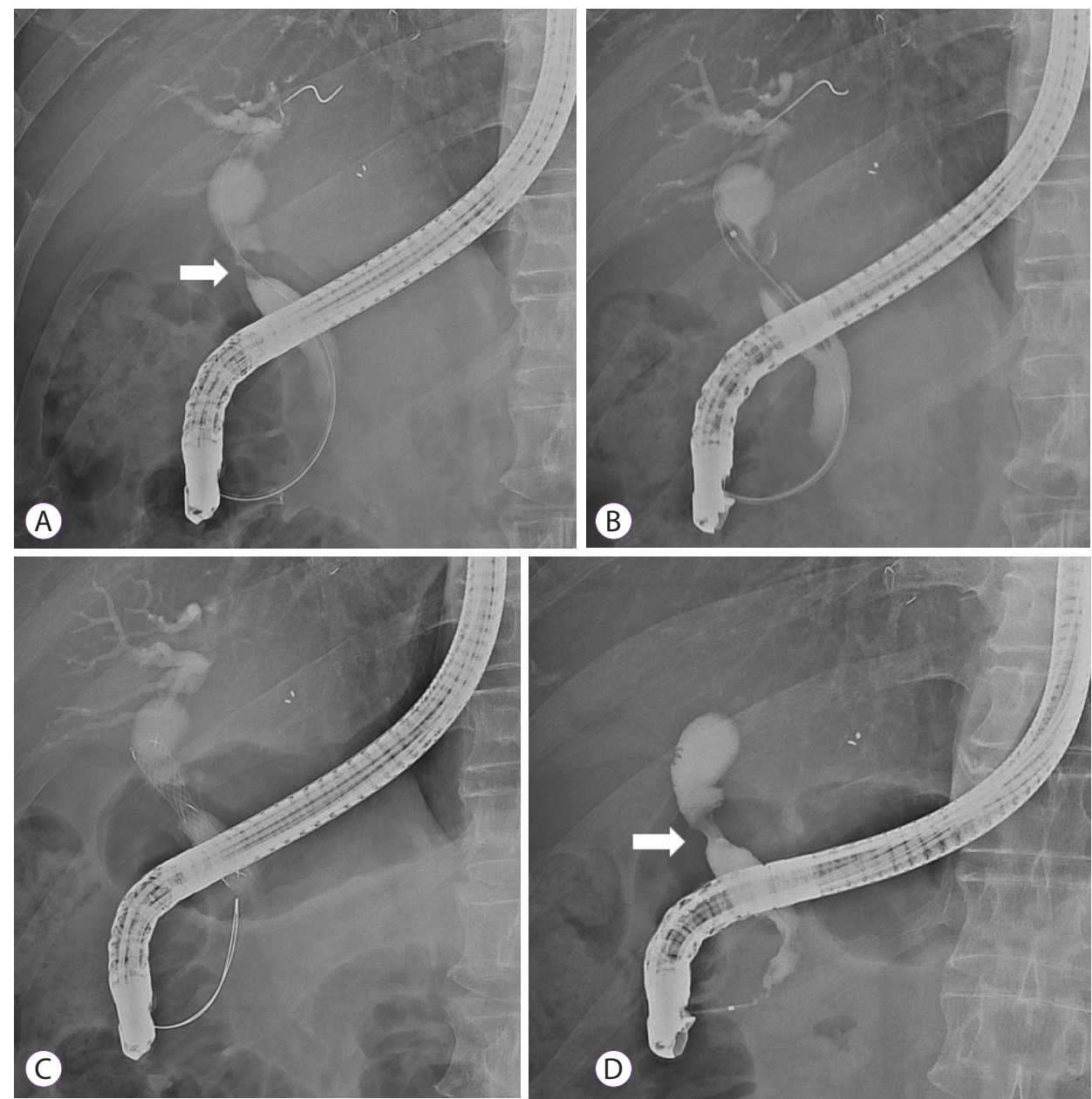

Fig. 1. Procedure to place fully covered self-expandable metal stent (CSEMS) for the treatment of anastomotic stricture (AS). (A) A guidewire was inserted through the AS (arrow) after the selective cannulation of bile duct. (B) Balloon dilatation of stricture was performed before cSEMS insertion. (C) Successful deployment of cSEMS was achieved. (D) AS resolution (arrow) was observed 6 months after cSEMS insertion. 
that there is a risk of perforation or bile leak at the anastomosis site when dilatation is attempted unreasonably within three months of LT. ${ }^{11}$ After stricture dilatation, delivery catheter of cSEMS can be passed easily through the AS and deployed optimally. (Fig. 1C).

Several methods of bile duct anastomosis such as end-toend, end-to-side, side-to-side exist; so, the type and level of AS also vary. Adjacent intrahepatic bile duct can be occluded after cSEMS insertion if AS occurs close to the proximal portion of extrahepatic bile duct. Thus, plastic stent insertion may be needed into the undrained bile duct after cSEMS insertion (Fig. 2).

\section{NEED FOR ENDOSCOPIC SPHINCTEROTOMY}

Martins et al. reported that eight cases of post-ERCP pancreatitis occurred in 16 patients who had cSEMS inserted without sphincterotomy. ${ }^{4}$ However, only one of the 14 patients who underwent sphincterotomy had pancreatitis. ${ }^{4}$ Theoretically, sphincterotomy increases the risk of cSEMS migration because the sphincter of Oddi loses the force to hold the cSEMS in place. Although some reports offer support against sphincterotomy promoting stent migration, ${ }^{16}$ these have focused on malignant biliary obstructions. It is hence difficult

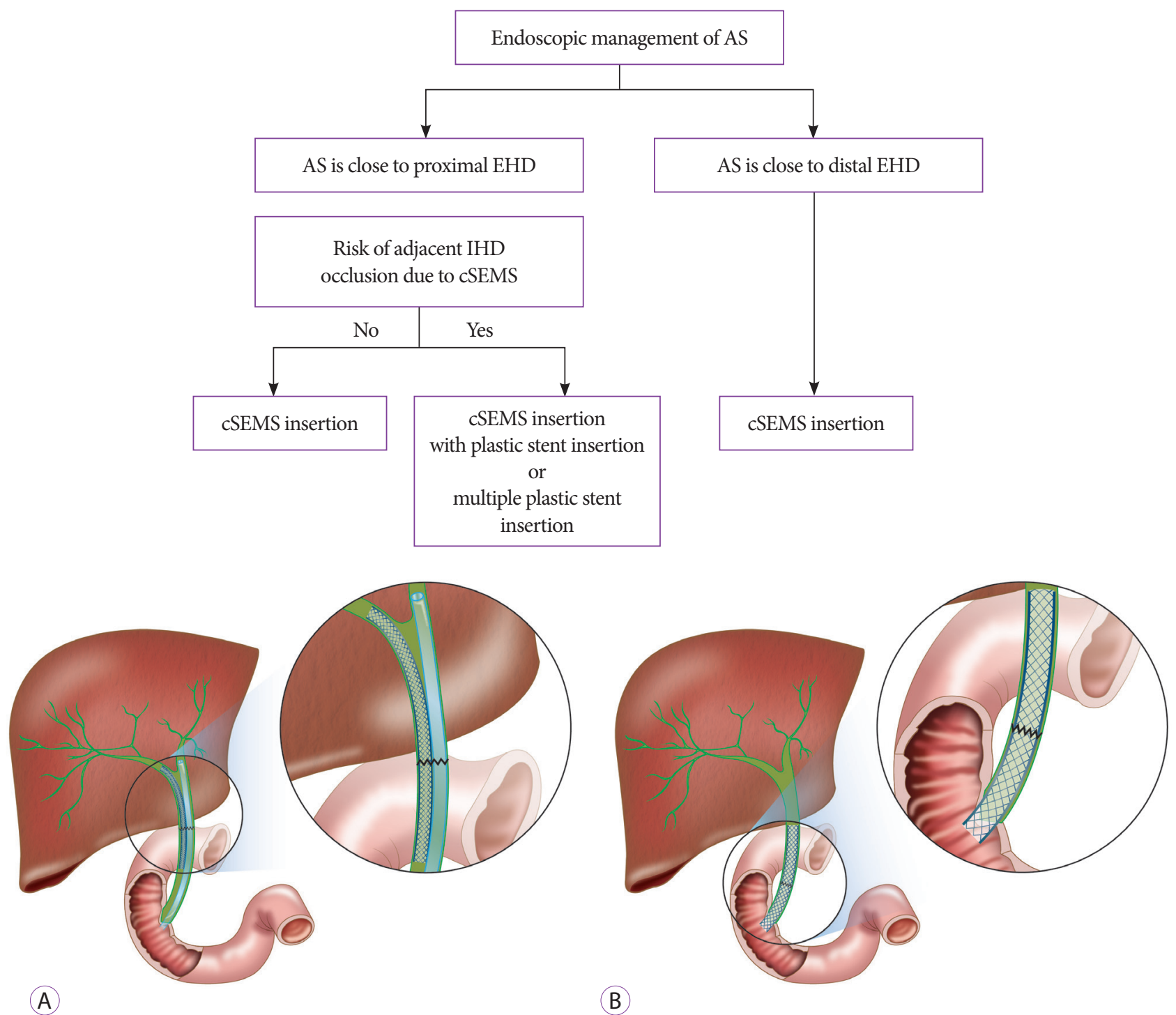

Fig. 2. The algorithm used for fully covered self-expandable metal stent (CSEMS) insertion according to the level of the anastomotic stricture (AS). (A) cSEMS and plastic stent insertion can be considered if AS is close to proximal extrahepatic bile duct (EHD). (B) Only cSEMS insertion is enough if AS is close to distal EHD. IHD, intrahepatic bile duct. 
to apply these findings to benign strictures such as AS. In addition, it should be kept in mind that sphincterotomy causes irreversible functional loss of the sphincter of Oddi, thus promoting stent occlusion due to duodenobiliary reflux or bacterial colonization. ${ }^{14}$

\section{DURATION OF CSEMS INDWELLING AFTER PLACEMENT}

In benign biliary strictures, prolonged indwelling of cSEMS can improve stricture resolution rates. ${ }^{17}$ The same results will likely be observed in AS following LT. However, the longer the indwelling period, the greater the risk of migration, and the higher the risk of secondary bile duct injury. Placement of cSEMS for unnecessarily long periods should be avoided because occlusion of the intrahepatic bile duct may occur around cSEMS insertion, resulting in bile stasis. ${ }^{18}$

\section{TREATMENT OUTCOMES (STRICTURE RESOLUTION \& STRICTURE RECURRENCE)}

In general, the resolution of the AS after cSEMS removal was checked using a cholangiogram (Fig. 1D). The success rate of AS resolution ranging from $53 \%$ to $100 \%$ was reported. ${ }^{4,719-23}$ In studies conducted before 2014, cSEMS dwelling usually lasted two to three months, whereas, in relatively recent studies, cSEMS lasted four to six months (Table 1). It is thought that the placement of cSEMS for longer periods helped achieve a higher resolution rate. According to a recent meta-analysis, there was no difference in the resolution rate of AS between use of cSEMS and MPS. ${ }^{13}$
Recurrence of stricture after cSEMS removal occurred in $17 \%-32 \%$ of cases. ${ }^{4,719-23}$ There was no statistically significant difference in the recurrence rate between the cSEMS and MPS groups. ${ }^{13,24}$ In addition, Martins et al. reported that in eight patients with recurrence, AS was resolved in six patients after 1 year of MPS dwelling. ${ }^{4}$ However, due to the small study group, it is difficult to consider this as the standard salvage management for AS. Further studies on this are therefore needed.

\section{MIGRATION OF cSEMS}

One of the most common complications of cSEMS treatment is stent migration. Although Kaffes et al. have reported the absence of migration with cSEMS, ${ }^{7}$ only 10 patients were involved in that study. With that result, it is hard to define cSEMS as the standard treatment for AS. Interestingly, migration does not mean treatment failure. Tal et al. reported that while migration occurred in 8 patients, 4 achieved AS resolution before the occurrence of migration. ${ }^{19}$ However, the migration of cSEMS may be associated with various adverse events and the requirement for additional procedures for stent repositioning or removal. Therefore, cSEMS migration is a problem that must be overcome.

\section{CONCLUSIONS}

So far, we have reviewed the usefulness of cSEMS as a treatment for AS that occurs after LDLT. The medical cost is lower when using cSEMS because the requirement for repeated ERCP procedures is less than that in MPS. Besides, the efficacy of cSEMS in terms of resolution and recurrence rates is similar to that of MPS. However, the decision should be

Table 1. Resolution, Recurrence and Migration Rate in Self-Expandable Metal Stent Insertion for Treatment of Anastomotic Stricture after Liver Transplantation

\begin{tabular}{|c|c|c|c|c|c|}
\hline Study & Patients, $n$ & $\begin{array}{l}\text { Duration of cSEMS } \\
\text { indwelling, mo }\end{array}$ & $\begin{array}{c}\text { Resolution of AS, } \\
n(\%)\end{array}$ & $\begin{array}{c}\text { Recurrence of AS, } \\
n(\%)\end{array}$ & $\begin{array}{c}\text { Migration of cSEMS, } \\
n(\%)\end{array}$ \\
\hline Martins et al. $(2018)^{4}$ & 30 & 6 & $25(83.0)$ & $8(32.0)$ & $3(10.0)$ \\
\hline Kaffes et al. $(2014)^{7}$ & 10 & 3 & $10(100.0)$ & $3(30.0)$ & $0(0.0)$ \\
\hline Tal et al. $(2017)^{19}$ & 24 & $4-6$ & $24(100.0)$ & $5(20.8)$ & $5(20.8)$ \\
\hline Poley et al. $(2012)^{20}$ & 23 & 5.5 & $14(61.0)$ & - & $6(26.1)$ \\
\hline Tarantino et al. $(2012)^{21}$ & 15 & 2 & $8(53.0)$ & $2(25.0)$ & $7(46.7)$ \\
\hline Sauer et al. $(2012)^{22}$ & 19 & $2-3$ & $6(75.0)$ & $1(17.0)$ & $8(42.1)$ \\
\hline Coté et al. $(2016)^{23}$ & 37 & 6 & $33(89.1)$ & $5(15.2)$ & $15(40.5)$ \\
\hline
\end{tabular}

AS, anastomotic stricture; cSEMS, fully covered self-expandable metal stent. 
carefully determined whether to perform stricture dilatation and sphincterotomy before cSEMS placement. The duration of cSEMS in position after placement has also not yet been determined. In addition, cSEMS migration is another hurdle to overcome. Therefore, studies on various types of cSEMS and management protocols will be needed in the future.

Conflicts of Interest

The authors have no financial conflicts of interest

ORCID

Dong Wook Lee: https://orcid.org/0000-0002-1029-9064

\section{REFERENCES}

1. Murray KF, Carithers RL Jr. AASLD practice guidelines: evaluation of the patient for liver transplantation. Hepatology 2005;41:1407-1432.

2. Durand F, Levitsky J, Cauchy F, Gilgenkrantz H, Soubrane O, Francoz C. Age and liver transplantation. J Hepatol 2019;70:745-758.

3. Stepanova M, Wai H, Saab S, Mishra A, Venkatesan C, Younossi ZM. The portrait of an adult liver transplant recipient in the United States from 1987 to 2013. JAMA Intern Med 2014;174:1407-1409.

4. Martins FP, De Paulo GA, Contini MLC, Ferrari AP. Metal versus plastic stents for anastomotic biliary strictures after liver transplantation: a randomized controlled trial. Gastrointest Endosc 2018;87:131.e1-e131.e13.

5. ASGE Technology Assessment Committee; Pfau PR, Pleskow DK, et al. Pancreatic and biliary stents. Gastrointest Endosc 2013;77:319-327.

6. Chaput U, Scatton O, Bichard P, et al. Temporary placement of partially covered self-expandable metal stents for anastomotic biliary strictures after liver transplantation: a prospective, multicenter study. Gastrointest Endosc 2010;72:1167-1174.

7. Kaffes A, Griffin S, Vaughan R, et al. A randomized trial of a fully covered self-expandable metallic stent versus plastic stents in anastomotic biliary strictures after liver transplantation. Therap Adv Gastroenterol 2014;7:64-71.

8. Girotra M, Soota K, Klair JS, Dang SM, Aduli F. Endoscopic management of post-liver transplant biliary complications. World J Gastrointest Endosc 2015;7:446-459.

9. Verdonk RC, Buis CI, Porte RJ, Haagsma EB. Biliary complications after liver transplantation: a review. Scand J Gastroenterol Suppl 2006;(243):89-101.

10. Pasha SF, Harrison ME, Das A, et al. Endoscopic treatment of anasto- motic biliary strictures after deceased donor liver transplantation: outcomes after maximal stent therapy. Gastrointest Endosc 2007;66:44-51.

11. Akhter A, Pfau P, Benson M, Soni A, Gopal D. Endoscopic management of biliary strictures post-liver transplantation. World J Metaanal 2019;7:120-128.

12. Facciorusso A, Rosca EC, Ashimi A, et al. Management of anastomotic biliary stricture after liver transplantation: metal versus plastic stent. Ann Gastroenterol 2018;31:728-734.

13. Visconti TAC, Bernardo WM, Moura DTH, et al. Metallic vs plastic stents to treat biliary stricture after liver transplantation: a systematic review and meta-analysis based on randomized trials. Endosc Int Open 2018;6:E914-E923.

14. Lee DW, Han J. Endoscopic management of anastomotic stricture after living-donor liver transplantation. Korean J Intern Med 2019;34:261268.

15. Rao HB, Prakash A, Sudhindran S, Venu RP. Biliary strictures complicating living donor liver transplantation: Problems, novel insights and solutions. World J Gastroenterol 2018;24:2061-2072.

16. Cui PJ, Yao J, Zhao YJ, Han HZ, Yang J. Biliary stenting with or without sphincterotomy for malignant biliary obstruction: a meta-analysis. World J Gastroenterol 2014;20:14033-14039.

17. Devière J, Nageshwar Reddy D, Püspök A, et al. Successful management of benign biliary strictures with fully covered self-expanding metal stents. Gastroenterology 2014;147:385-395; quiz e15.

18. Tsujino T, Sugawara Y, Omata M. Management of biliary strictures after living donor liver transplantation. Gastrointest Endosc 2009;70:599-600;author reply 600-601.

19. Tal AO, Finkelmeier F, Filmann N, et al. Multiple plastic stents versus covered metal stent for treatment of anastomotic biliary strictures after liver transplantation: a prospective, randomized, multicenter trial. Gastrointest Endosc 2017;86:1038-1045.

20. Poley JW, Cahen DL, Metselaar HJ, et al. A prospective group sequential study evaluating a new type of fully covered self-expandable metal stent for the treatment of benign biliary strictures (with video). Gastrointest Endosc 2012;75:783-789.

21. Tarantino I, Traina M, Mocciaro F, et al. Fully covered metallic stents in biliary stenosis after orthotopic liver transplantation. Endoscopy 2012;44:246-250.

22. Sauer P, Chahoud F, Gotthardt D, et al. Temporary placement of fully covered self-expandable metal stents in biliary complications after liver transplantation. Endoscopy 2012;44:536-538.

23. Coté GA, Slivka A, Tarnasky P, et al. Effect of covered metallic stents compared with plastic stents on benign biliary stricture resolution: a randomized clinical trial. JAMA 2016;315:1250-1257.

24. Lee DW, Jo HH, Abdullah J, Kahaleh M. Endoscopic management of anastomotic strictures after liver transplantation. Clin Endosc 2016;49:457-461. 